\title{
LE DROIT COMPARÉ ET L'UNIFICATION DU DROIT PRIVÉ DES PAYS LATINS
}

\author{
par
}

\section{O. Martins Gomes}

Professeur de Droit International Privé à la Faculté de Droit de l'Université du Paraná, Brésil.

- Membre du Comité Brésilien pour le Vème Congrès International de Droit Comparé, de Bruxelles.

- Représentant de l'Institut des Avocats Brésiliens au Conseil de l'Union Internationale des Avocats, à Paris.

1 - La liste des matières du Ve. Congrès International de Droit Comparé, organisé par l'Académie de Droit Comparé, de Bruxelles, distingue entre autres sujets d' extrème importance celui dont se revêt le droit comparé comme moyen de recherche des matières susceptibles d'être unifiées sur le plan international. (Sec. I, G-1). Les auteurs ayant été priés de choisir un sujet de leur droit national, une certaine difficulté peut surgir quand il s'agit de concilier la ligne de conduite qu'on est tenu de suivre avec un sujet d'ordre général tel celui qui a été choisi. La place qu'y tient le droit brésilien est celle d'un élément de comparaison, à peine, face à d'autres systèmes juridiques auxquels l'unissent des liens de parenté, un même degré de civilisation et un même niveau culturel joint à un même état economico-social, comme le voulait E. LAMBERT en vue de la prétendue application d'un droit commun. 
Dans les pays neufs comme ceux d'Amérique, c'est surtout aux dépends de l'expérience acquise par les pays hautement civilisés que s'est formé le droit. Les recherches qui s'effectuent dans le domaine du droit comparé ont dans ces cas-là un rôle préponderant et, selon G. TARDE, la législation, sauf certains aspects déterminés qui lui sont propres, se fonde sur le principe de l'imitation.

Le Brésil était jusqu'en 1822 une colonie portugaise régie par le système législatif de la métropole. Le droit privé prenait sa source dans le droit romain, origine commune du droit continental européen, sous son aspect le plus ample du "usus modernus juris romani", c'est à dire, du droit romain réavivé par l'interprétation donnée à ses textes par les glosateurs et post-glosateurs du Moyen-Age.

Une fois obtenue la consolidation de ses lois civiles après son indépendance et grâce au travail herculéen de l'ingénieux juriste TEIXEIRA DE FREITAS, l'une des plus hautes expressions de la pensée juridique surgie dans les Amériques, le Brésil s'attache au long travail d'élaborer son code civil, promulgué en 1916 et toujours en vigueur, bien qu'ayant en bonne partie subi les modifications de lois postérieures. Ce code civil a souffert l'influence du droit continental, mais il faut, toutefois, souligner sa plus grande afinité avec les législations analogues des pays latins. II convient de faire remarquer que, bien qu'étant constitué d'une fédération de provinces autonomes ou états membres, par son organisation politique, le pouvoir de légiférer au Brésil, en matière de droit civil, commercial, pénal, électoral, aeronautique, travailliste et de la procédure, est de la compétence exclusive de l'Union Fédérale et cela pour tout le pays.

2 - Le présent travail a pour but d'étudier la possibilité d'unification du droit privé du groupe des pays latins; de ces peuples d'origine commune, d'un même sang, qui ont subi pareillement les influences variées des mêmes faits, de la réligion, des idées morales, des coutumes et de la tradition.

D'aprés GUGLIELMO FERRERO, les caractéristiques essentielles de l'esprit latin sont l'ordre, l'harmonie et la me- 
sure, alliés à l'idéalisme, à la propension à la philosophie, à la recherche scientifique, au culte de la beauté et au sentiment de la pitié. Suivant ANDRÉ SIEGFRIED, les nations de ce bloc sont latines parce qu'elles parlent des langues romaniques, parce qu'elles sont l'instrument d'une certaine expression de pensée qui correspond de façon indiscutable à une civilisation dont la Méditerranée est le berceau et qui a su s'imposer lors de la lumineuse éclosion de la Renaissance.

Les siècles ont vu s'accentuer à chaque pas la puissante influence du génie gréco-romain, visible dans toutes les manifestations importantes de la grandeur de l'esprit, de la philosophie, des sciences, de la politique, du droit et des arts.

3 - C'est grâce à l'initiative du gouvernement brésilien que s'est réuni en 1951, à Rio de Janeiro, le I Congrés de l'Amérique Latine. II a approuvé, entre autres délibérations, le projet-base brésilien reconnaissant l'importance du rôle historique joué par les peuples d'origine latine relativement à l'évolution de la pensée et au développement de la civilisation mondiale et il a affirmé l'existence des liens que les unissent pour la sauvegarde d'un patrimoine comun. II a, en conséquence recommandé ces objetifs aux gouvernements et aux peuples latins dans l'intérêt de la consolidation de l'Union Latine à savoir: a) assurer la sauvegarde des principes sur lesquels repose la civilisation latine et de la façon de vivre et de penser qui constitue ses caractéristiques essentielles; b) favoriser l'entretien des liens spirituels et l'échange intellectuel entre les peuples latins.

Lors de l'approvation par le Congrés de Rio de Janeiro de la thése présentée par la délegation brésilienne, celui-ci a reaffirmé sa foi dans les valeurs spirituelles qui forment l'essence de la civilisation humaniste et chrétienne des nations. A propos de l'objetion posée par le délégué du Mexique quant à l'expression "chrétienne", objection sur le principe constitutionel de la liberté de culte, et à la proposition de la substituer par l'expression "méditerranéenne", le juriste brésilien RAUL FERNANDES expliqua que l'idée chrétienne n'impliquait aucune affirmation de principe religieux mais trouvait son fonde- 
ment dans le fait que le christianisme a été une des sources historiques de la latinité, qui en est imprégnée de façon intégrale.

II faut, entre autres, souligner la proposition votée par le Congrès qui recommande une étroite collaboration entre l'Union Latine et l'Organisation des Nations Unies, ainsi que la proposition qui crée un INSTITUT DE DROIT COMPARÉ à Paris, siège du Secretáriat Général de l'Union Latine .

Le groupe latin comprend en Europe la France, l'Italie, l'Espagne, la Roumanie, le Portugal; en Amérique, tous les pays qui composent l'Amérique Latine et en Asie les Iles Philippines. Excepté la Roumanie, le bloc européen jouit de continuité territoriale, de même que l'ensemble des pays américains, mais il n'en est pas de même avec les Iles Philippines.

4 - Dans le but de contourner les difficultés DE DIVERSITATE LEGUM entre les différents systèmes législatifs dans le domaine du droit privé, certaines conventions internationales réalisent ce que RAOUL DE LA GRASSERIE appelle l'unification PROVISOIRE, qui consiste en un même corps de règles en vue de dirimer les conflits des legislations, soit un superdroit destiné à donner une solution aux problèmes interspaciaux et permettant l'application du droit substantif le plus approprié à chaque cas. L'unification DÉFINITIVE des lois des différentes nations se ferait plus tard. D'abord celle des lois commerciales et civiles, ensuite celle des lois politiques et administratives.

C'est ainsi que le Code Bustamante, voté à La Havane en 1928 et observé dans quinze pays américains est déja un exemple de l'unification provisoire. Il en va de même pour ce qui concerne les normes de conflit qui ont été approuvées aux Conférences de La Haye de la fin du siécle dernier et du début de celui-ci. C'est ainsi que la Convention de Genève de 1930, d'où surgit la "Loi Uniforme" concernant les lettres de change et les billets à ordre, est l'exemple de l'unification définitive.

Ces divers essais constituent un pas de géant réalisé vers le but proposé de l'unification, et cela tout en tenant compte des réserves faites par les pays qui ont signé ces deux conven- 
tions. Faisons aussi remarquer que toujours à Genève fut élaborée une autre convention ayant pour but de régler certains conflits de lois dans cette même matière d'effets de commerce.

R. DEMOGUE distingue trois catégories d'unification: l'imitation, quand un état se limite à copier les lois d'un autre; l'adoption pure et simple par un état des lois d'un autre, et la catégorie des concessions réciproques entre divers états. C'est cette dernière catégorie qui a, en général, reçu les suffrages lors des conférances internationales.

FR. CONTUZZI, cité par I. P. MARINHO, fait aussi remarquer que dans les pays imprégnés d'une même culture juridique, les matiêres ou questions en cause se revêtent d'un caractère d'universalité tout comme celles qui découlent du droit commercial et en particulier du droit maritime. C'est ce caractère d'universalité qui peut alimenter de plus près encore l'espoir de l'unification souhaitée. On a d'ailleurs réussi à la transposer sur plan pratique, comme le prouve les conventions qui s'ocuppent du droit du change, qui, sous son aspect abstrait, ne suscite pour ainsi dire que des problemès techniques.

La branche du droit dont l'unification offre le plus de difficultés est le droit de la famille parce qu'il dépend davantage des coutumes locales, des croyances, des préjugés, des traditions parce qu'elle a ses racines dans une façon de sentir, de penser et d'agir qui est particulière à chaque peuple et qu'elle entraine les législateurs à envisager certaines institutios juridiques de manière différente et à les transformer en préceptes d'ordre public.

Sans parler de la monogamie, forme d'organisation de la famille adoptée dans les pays occidentaux, on s'aperçoit que le divorce n'est pas admis dans toutes ces législations, qui conservent cependant entre elles de si forts liens d'affinité, qui ont été engendré par de semblables influences au cours des étapes de leur formation et de leur progrés. Les conférences de la Haye sont arrivées, dans leurs diverses réunions périodiques, à ce que beaucoup de nations se mettent d'accord pour ce qui concerne le mariage, la séparation, la tutelle et la curatelle. 
5 - Au Congrès de l' "Internacional Bar Association", à Madrid en 1952, auquel l'auteur a eu l'honneur de prendre part comme membre de la délégation brésilienne, Mr. MARIO MATTEUCCI, Sécrétaire Général de l'Institut International pour l'Unification du Droit Privé, dont le siège est à Rome, expliqua que l'institut traverse les différentes étapes décrites par le professeur H. C. GUTTERIDGE,, de l'Université de Cambridge, dans son livre "Comparative Law", publié en 1954. Parmi elles nous distingons l'étape préparative qui étudie les raisons qui rendent la comparaison nécessarie, d'on la convenance d'installer dans chaque pays un Comité National de Droit Comparé comme celui de Rio de Janeiro, affilié à l'Unesco, qui fonctionne sous la présidence de l'éminent professeur Haroldo Valladão. Vient ensuite l'étape énumérative, qui consiste à faire esquisser le droit uniforme par des juristes et des techiniciens internationaux. Puis, c'est l'étape opérative, qui comprend tous les travaux exécutés en vue de l'adoption du droit uniforme esquissé et qui peut être divisée en deux phases: l'une est ne consultation préliminaire faite auprès des organismes publiques intéressés à l'unification, l'autre la présentation d'un avant-projet aux gouvernaments respectifs qui pourront alors écouter les opinions des juristes et des professeurs à travers leurs sociétés et leurs académies, en vue de son adoption par ces gouvernements. Enfin, l'étape finale, ou a lieu la présentation du projet à une conférence diplomatique, précédée d'une réunion des Comités préparatoires et composée des représentants des gouvernements en vue de faire une analyse soigneuse des changements sugérés, facilitant de cette façon le travail qui sera fait au cours de la conférence. Dans ce plan GUTTERIDGE rappelle que la conférence n'est pas l'unique moyen qu'ont les gouvernements d'arriver à l'adoption d'un droit uniformisé. Les lois-types permettent d'atteindre un certain degré d'uniformité indépendamment d'accord international, comme on voit dans les pays scandinaves. Ce système, où les lois-types sont mises en pratique dans divers pays, est d' exécution plus facile là où les législations sont ou plus ou moins 
ressemblantes. En pratique, on recontre souvent dans le droit mercantil, surtout là où l'on traite de la vente des marchandises, des clauses-types qui facilitent l'échange commercial. 6 - C'est en proie à une explicable timidité que l'auteur se permet de suggérer dans ce modeste exposé d'ordre général et fondamental, qui n'entre dans acune particularité, l'unification du droit privé des pays latins.

ARMINJON, grande autorité en droit international privé, classifie sept principaux systèmes législatifs du monde: celui du droit commun ("Common Law"), des pays anglo-saxons; celui qui s'inspire du système français, qui s'étend à de nombreuses parties de l'Europe, aux territoires français d'outremer et à l'Amérique Latine; celui qui dérive du droit allemand et qui inclu le droit civil suisse, imité par les codes civils ture, japonais et siamois; le système scandinave; le russe; islamique et l'hindou. Le groupe latin fait évidemment partie du système d'inspiration française, au sein de cette classification, bien que autres pays en participent également.

Il y a un peu plus d'un siècle, à peine, que l'institution de chairs de droit comparé dans les écoles supérieures d'Europe, notamment à l'Université de Paris, ont donné un élan à la fois visible et ordonné au droit comparé. A cette même époque, croissait, grâce aux magnifiques travaux de juristes européens comme Savigny, Lainé, Fiore et Laurent, l'importance du Droit International Privé.

L'affinité du droit comparé et du droit international privé est manifeste. Plusieurs poits leur sont communs puisque tous deux tendent au même but: la nécessité d'une colaboration internationale en matière de droit privé, selon la pensée de $\mathrm{H}$. C. GUTTERIDGE, qui fait encore remarquer: "Tout comparatiste doit nécessairement connaitre le droit international privé sinon il ne pourra jamais se rendre compte dans quelle mesure les différences des droits constituent un obstacle aux rapports internationaux". ("Le Droit Comparé" trad. franc. de René David et ses élèves, Paris, 1953, pages 65 et 66 ).

Que l'on considère le droit comparé comme une science en lui reconnaissant son autonomie, qu'on le considère comme une 
méthode ou comme une technique d'application de la méthode comparative, ce n'est, en vérite, que la recherche juridique qui importe pour la plus grande satisfaction des aspirations des comparatistes.

L'auteurx anglais cité plus haut remarque que, quoi qu'il en soit, la méthode comparative, (c'est son expression favorite), est à l'épreuve. Nous vivons perpétuellement en état d'experience et l'énumération et la classification de ses fonctions possibles ne peuvent pour cette raison être considérées comme définitives (pg. 28).

Le droit comparé est le fondement de la méthode, l'ensemble des moyens théoriques et pratiques destinés à atteindre le but visé

La these de l'unification du droit privé des pays latins peut sembler teméraire aujourd'hui, mais une fois realisée elle laissera à découvert les vastes horizons jusqu'où elle pourra s'étendre, et cela dans l'interêt d'une plus grande et meilleure réalisation de la communauté du droit entre les peuples civilisés de l'univers. 